\title{
Geografías de la razón: de los lugares de producción del conocimiento ${ }^{1}$
}

\author{
Magaly Vega Rodríguez² \\ Pontificia Universidad Javeriana
}

Recibido: 13 de noviembre de 2009 A Aprobado:3 de mayo de 2010

\section{esumen}

Partiendo de los análisis de Doreen Massey y de Santiago Castro-Gómez, se presenta una reflexión en torno a las geografías de la razón, que intenta mostrar la importancia de pensar los lugares desde los que se enuncia el conocimiento y lo que tienen de localizados los sistemas de enunciabilidad, de funcionamiento, formación y transformación de los discursos. Para ello, se ${ }^{3}$ divide la presentación en cuatro partes. Primero, se hace una breve presentación del cuestionamiento foucaultiano de la producción de los discursos. Segundo, se retoma los aportes de Massey a propósito de una geografía de la producción del conocimiento. Se presenta la tesis de Castro-Gómez sobre"la hybris del punto cero"y la localización de los saberes. Finalmente, se apunta a una reflexión que articula los aportes de nuestros autores en la necesidad de pensar los lugares de enunciación del conocimiento como fundamento de la afirmación de la multiplicidad epistémica.

Palabras clave: producción del conocimiento, geografía, lugar de enunciación, discursos, hybris del punto cero.

1 Informe de resultados parciales de la investigación "Multiplicidad epistémica" financiada por Colciencias y el Instituto Pensar de la Pontificia Universidad Javeriana.

2 Filósofa. Candidata al título de Maestría en Filosofía de la Pontificia Universidad Javeriana. Joven Investigadora del Instituto de Estudios Sociales y Culturales PENSAR de la Javeriana, gracias a una beca otorgada por Colciencias. Investigadora del grupo Filosofía, Cultura y Globalización (A) de la Facultad de Filosofía y Humanidades de la Universidad de La Salle e investigadora del Centro de Investigación en Hábitat, Desarrollo y Paz -CIHDEP- de la misma universidad. Correo electrónico:magaly.vega@javeriana.edu.co

3 "La crítica es el movimiento por el cual el sujeto se atribuye el derecho de interrogar a la verdad acerca de sus efectos de poder y al poder acerca de sus discursos de verdad; la crítica será el arte de la inservidumbre voluntaria, de la indocilidad reflexiva. La crítica tendría esencialmente como función la de sujeción en el juego de lo que se podría denominar, con una palabra, la política de la verdad" (Foucault, 2003, pp. 10-11). 


\section{Geography of the reason: from the places of knowledge production}

\section{Abstract}

Based on the analysis of Doreen Masey and those of Santiago Castro-Gómez, we will show a reflection on the geographies of the reason that will attempt to show the importance of thinking about the places from which knowledge is expressed and what they have in localized systems of statements, operating, training and transformation of discourses. To do this we divide the presentation into four parts. First, we will make a brief presentation of Foucault's questioning of the production of discourse. Second, we will take the contributions by Masey about a geography of knowledge production, then we will present the thesis by Castro-Gómez on "The hubris of the zero point" and the location of knowledge, and finally we will aim to reflect the contributions of our authors on the need to think about the places of enunciation of knowledge as a basis for the assertion of the epistemic variety.

Key words: knowledge production, geography, site of enunciation, speeches, hubris of zero. 


\section{Géographie de la raison: des lieux de production de la connaissance}

\section{Résumé}

En partant des analyses de Doreen Masey et de Santiago Castro-Gómez, nous présenterons une réflexion autour des géographies de la raison qui tentera de montrer l'importance de penser les lieux depuis lesquels s'énonce la connaissance et ce que les systèmes d'énonciabilité, de fonctionnement, de formation et de transformation des discours ont de localisé. Pour ce faire, nous diviserons la présentation en quatre parties. D'abord, nous ferons une brève présentation du questionnement foucaultien de la production des discours. La seconde partie reprendra les apports de Masey à propos d'une géographie de la production de la connaissance. Ensuite, nous présenterons la thèse de Castro-Gómez sur «l'hybris du point zéro» et la localisation des savoirs et enfin, nous viserons une réflexion qui articule les apports de nos auteurs autour de la nécessité de penser les lieux de l'énonciation de la connaissance comme fondement de l'affirmation de la multiplicité épistémique.

Mots-clé: production de la connaissance, géographie, lieu d'énonciation, discours, hybris du point zéro. 


\section{Introducción}

Los griegos decían que la hybris es el peor de los pecados, pues supone la ilusión de poder rebasar los límites propios de la condición mortal y llegar a ser como los dioses. La hybris supone entonces el desconocimiento de la espacialidad y es por ello un sinónimo de arrogancia y desmesura

Santiago Castro-Gómez

La tradición filosófica y epistemológica en general ha entronizado la producción especializada del pensamiento europeo occidental a tal punto que es conocida y enseñada como la Historia del Pensamiento Universal, creando de paso ciertos patrones de juicio a los cuales debe ser sometida toda nueva producción del pensamiento que quiera entrar en esa tradición. Las reflexiones enmarcadas en la filosofía de la sospecha, la teoría crítica y el posestructuralismo, entre otras, son muestra de la preocupación y el debate que ha despertado este tipo de universalización del pensamiento, que lejos de ser una representación universal, en el sentido estricto del término, ha elevado a este plano las producciones locales de Europa, y en los últimos años, de EE. UU., pretendiendo que toda expresión del hombre (ya sea americano, africano, asiático, australiano o europeo) se encuentre representado en lo que Occidente (Europa y EE. UU.) ha dado en Ilamar "universal" (por ejemplo, los derechos humanos).

La modernidad instituyó la objetividad y la universalidad como requisitos fundamentales de todo conocimiento que quisiera ser tenido por científico, y con esto, por válido y legítimo. Sin embargo, como ha sido anotado por la mayoría de pensadores del siglo XX, el conocimiento científico moderno desconoce los procesos de producción que posibilitan la emergencia de discursos inscritos en esta tradición y, aún más, este tipo de discursos legitiman, refuerzan e incluso crean estrategias de dominación sobre poblaciones, mercados, saberes, etc. Esto es lo que describe la relación poder/conocimiento explicitada por Foucault y tenida en adelante como supuesto de las investigaciones en ciencias sociales. Michel Foucault desarrolló, como ningún otro pensador del siglo XX, un análisis de la relación entre conocimiento e 
historia hasta la Edad Moderna, dejando sentadas las bases para una revisión crítica de la verdad, del conocimiento, del poder, que pone en entredicho su carácter histórico y nos pone frente a sus condiciones de producción.

Todo el diagnóstico de Foucault está basado en la historicidad de las producciones discursivas, pero está desprovisto de un análisis de la espacialidad. En el escrito De los espacios otros (1967), Foucault (1984) intenta pensar el espacio aduciendo que "la inquietud actual concierne fundamentalmente al espacio, sin duda mucho más que al tiempo; el tiempo no aparece probablemente sino como uno de los juegos de distribución posibles entre los elementos que se reparten en el espacio" (p. 2).Pero el espacio, según afirma el autor, no ha alcanzado un nivel de desacralización práctica, ni teórica totalmente.

La obra de Foucault, en lo que concierne a la producción discursiva, se empeña en demostrar la historicidad y contingencia de los discursos para desnaturalizar la necesidad de proyectos modernos como la llustración y el progreso histórico. Sin embargo, y aún después de haber hecho la anotación sobre la necesidad de pensar el espacio, su diagnóstico sobre conocimiento, como se dijo más arriba, está desprovisto de la reflexión sobre la espacialidad de la producción de discursos. Massey (2005), en cambio, desarrolla su reflexión sobre un eje espacial, se preocupa por desmontar todas las concepciones tradicionales del espacio que lo subordinan al tiempo. Sin embargo, nos interesan aquí particularmente las reflexiones que desarrolla en torno a la producción del conocimiento y a lo que llama "las geografías del conocimiento".

Partiendo de los análisis de esta geógrafa y de los que, desde Foucault y los estudios poscoloniales, desarrolla Santiago Castro-Gómez, presentaré una reflexión en torno a las geografías de la razón que intenta mostrar la importancia de pensar los lugares desde los que se enuncia el conocimiento y lo que tienen de localizados los sistemas de enunciabilidad, de funcionamiento y de formación y transformación de los discursos. Para ello, dividiré la presentación en cuatro partes. En primera instancia haré una breve presentación del cuestionamiento foucaultiano de la producción de los discursos que servirá de preámbulo para las siguientes cuestiones. La segunda parte retomará los aportes de Massey a propósito de una geografía de la producción 
del conocimiento. En seguida, presentaré la tesis de Castro-Gómez sobre la hybris del punto cero y la localización de los saberes; y por último, apuntaré a una reflexión que articule los aportes de nuestros autores en la necesidad de pensar los lugares de enunciación del conocimiento como fundamento de la afirmación de la multiplicidad epistémica.

\section{De la contingencia de la verdad y el control de los discursos}

En la modernidad, el conocimiento era tenido por necesario; el fin último del hombre era alcanzar la máxima expresión del conocimiento, que algunos encontraban en la ciencia y otros en la filosofía. La verdad estaba dada en las cosas en sí y era tarea del espíritu humano, de la conciencia, desentrañar esa verdad, alcanzarla. No se trataba de interpretar el mundo, sino de encontrar el camino más preciso a la cosa en sí. La verdad reposaba eternamente hasta ser descubierta y alcanzada por el hombre.

Según nos dice Foucault, con la modernidad aparece el hombre como objeto de estudio y sujeto de representación. Esto da al hombre un dominio mayor sobre su ejercicio discursivo, pero, aún así, siguen operando las instancias últimas de legitimación. Para entender las condiciones de posibilidad de los discursos modernos, Foucault pone de manifiesto la matriz saber-poder que determina las prácticas discursivas en la Modernidad.

Con el surgimiento del hombre como objeto de estudio, nacen las llamadas ciencias humanas que tratarán de determinar -para controlar- los ámbitos de la vida del hombre (Foucault, 1968). Foucault llama a estas prácticas discursivas tecnologías, ya que son saberes prácticos que permiten un dominio técnico sobre ciertos ámbitos de la vida. La sociología, la antropología, la psicología, la historia, pedagogía, etc., se constituyen en saberes expertos que articulan la praxis social y dan los parámetros para su legitimación. La modernidad instaura la producción artificial de una sociedad desde discursos expertos cuya tarea será controlar al hombre desde la creación de seguridades ontológicas del tipo del Estado, la familia, la policía, etc. Instituciones, discursos y subjetividades van fortaleciendo una voluntad de verdad que 
refuerza el proyecto de dominio de los saberes expertos. No cabe hablar aquí de la verdad como instancia última del conocimiento, Foucault deja claro que la verdad es una función que las diferentes voluntades (los distintos proyectos de dominio de las sociedades) se disputan constantemente. Lo que conocemos en cada época como la verdad es una producción vigilada y determinada por voluntades arbitrarias.

Es necesario que estas disciplinas se regulen también, pues hay que evitar que el azar domine las prácticas discursivas (Foucault insistirá en que ésta es la naturaleza de los discursos: son acontecimiento, azar, contingencia). En palabras de Foucault (1992): "en toda sociedad la producción del discurso está a la vez controlada, seleccionada y redistribuida por un cierto número de procedimientos que tienen por función conjurar los poderes y peligros, dominar el acontecimiento aleatorio y esquivar su pesada y temible materialidad" (p. 12).

Un pensamiento crítico ${ }^{4}$ para Foucault consistiría entonces en reconocer en cada discurso las condiciones que hicieron posible su emergencia, tener en cuenta que se inscribe en un sistema de enunciabilidad que define su forma de aparición, en un sistema de funcionamiento que define su modo de actualidad y en un sistema de formación y transformación que define las reglas de su subsistencia y modificación (Foucault, 1970, pp. 220-221). Estos sistemas no son abstractos y generales, sino que son condicionados por variables como lugar de emergencia, raza, género, cultura, entre otros.

El proyecto foucaultiano no intenta, como la tradición filosófica lo hizo, buscar legitimación a las formas de conocimiento y de poder que instituyen una sociedad. Por el contrario, trata de determinar cuáles son las condiciones de aceptabilidad de un sistema de dominio en una sociedad. En este proceso se desnaturalizan los procesos para hacer ver que no son necesarios históricamente, que no son el efecto necesario del pasado monumental, sino que son producidos.

4 Para sustentar y mantener el poder sobre una población y territorio definidos, la organización estatal optó, entre otras estrategias, por unificar la identificación de los habitantes con el territorio. La identidad nacional debe entenderse en este sentido como un conjunto de discursos y prácticas que afianzan y legitiman el ejercicio del poder en un territorio determinado. 
Ahora bien, desnaturalizar los discursos que sostienen un sistema de dominio debe pasar también por hacer evidente que la localización de dichos discursos instituye jerarquías y delimitaciones que resaltan el carácter de producidos de esos discursos constituidos en conocimiento experto. Para tratar la cuestión de la espacialidad en el conocimiento retomaré los aportes de Doreen Massey.

\section{Geografías de la producción del conocimiento}

Para la geógrafa Doreen Massey (2005), más que historizar los discursos y proyectos de la modernidad, es necesario espacializarlos de manera que se descentre a Europa del lugar que ocupa en las representaciones modernas. Esto permitiría pensar la historia europea como sólo una más de las múltiples historias locales. La historia europea y sus proyectos civilizatorios se han autorrepresentado como universales, haciendo que cada expresión de lo humano deba ser mirada bajo sus códigos para poder recibir el adjetivo de universal.

Espacializar la modernidad, además de negar la pretendida universalidad de los discursos modernos sobre el hombre, la historia y la sociedad, permite revelar la posicionalidad geográfica de aquellos discursos y, con esto, entender la espacialidad de la producción del conocimiento en sí mismo. La espacialización, entonces, es otra vía para evidenciar la relación entre poder y conocimiento (evidenciada por Foucault a partir de la historización) y con ella, la emergencia de la geografía del poder.

Massey (2005) presenta los Estados-nación como una forma de expresión de lo que llama la geografía del poder. Para Massey, la forma de organización en Estados-nación, típica de la modernidad, permite que los grupos poblacionales se integren por la "pertenencia" a un territorio delimitado y diferenciado de los demás por separación ${ }^{5}$. Pensar el espacio como sólidamente dividido

5 En el ámbito de la cartografía, por ejemplo, la perspectiva se adopta como el punto cero de observación: "esto revoluciona por completo la práctica científica de los cartógrafos. Al tornarse invisible el lugar de observación, el centro geométrico ya no coincide más con el centro étnico. Por el contrario, los cartógrafos y navegantes europeos, dotados ahora de instrumentos precisos de medición, empiezan a creer que una representación hecha desde el centro étnico es precientífica, pues queda vinculada a una particularidad 
no es algo natural ni necesario, sino producto del proyecto de la modernidad. Contrario al discurso moderno, la especificidad cultural, según dice Massey, no se halla en el distanciamiento espacial, sino en las interacciones que se articulan en los lugares y más allá de ellos.

Representar el espacio a partir de configuraciones temporales, como tradicionalmente se hace, evidencia que las concepciones de tiempo y espacio son claves para construir un sistema particular de poder/conocimiento. La concepción temporal del espacio es generalmente usada, dentro de ese sistema, para incrementar las distancias entre comunidades que incluso coexisten en un mismo espacio. Así, por ejemplo, al momento de la colonización, se temporalizó la coexistencia de comunidades indígenas, negras, criollas y españolas en el mismo espacio geográfico; en el sistema de poder/ conocimiento (de dominación) los negros y los indígenas aparecían como vestigios de un pasado ya superado por criollos y españoles:

Aunque en el presente tengamos experiencias de una gran cantidad de sociedades simultáneas en el espacio, no todas estas sociedades son simultáneas en el tiempo. Bastará con observar comparativamente, siguiendo el método analítico, para determinar cuáles de esas sociedades pertenecen a un estadio inferior de la escala evolutiva" (Castro-Gómez, 2005a, p. 33).

Este distanciamiento, según Massey (2005), tiene el efecto de minimizar la vigencia actual de la diferencia.

Es por esto que para Massey es evidente la existencia de una geografía de la producción del conocimiento que espacializa ciertos lugares donde se llevan a cabo las representaciones. Esta espacialización establece las funciones de conocedor y conocido e instaura la distancia necesaria entre ellos para mantener dentro de los márgenes la producción del conocimiento. Esto inhabilita a lo que es definido como conocido para conocerse y para producir conocimiento.

cultural específica. La representación verdaderamente científica y objetiva es aquella que puede abstraerse de su lugar de observación y generar una 'mirada universal' sobre el espacio" (Castro-Gómez, 2005a, pp. 59-60). 
La geografía de la producción del conocimiento se produce no sólo conceptual sino también materialmente, lo que se evidencia en la generación de la distancia entre el conocedor y lo conocido. En palabras de Massey (2005): "the spatial structures of knowledge production which assume a radical gap between knower and known are precisely ones through which equation between representation and spatialisation can be confirmed" (p. 75). En este marco, el prestigio del conocedor se deriva de su espacialidad y ésta misma lo legitima para la producción de representaciones sobre lo conocido. Las representaciones del conocedor colonizan el lenguaje y son tomadas como las únicas fuentes de conocimiento. Nuevamente vale anotar el caso de la colonización. Europa ocupaba el centro del sistema de representación desde el cual enunciaba a las comunidades colonizadas, determinando lo que éstas debían ser en este sistema. Para Massey, estos discursos, antes que ser descriptivos e informar sobre cómo es el mundo, son normativos y representan una imagen de cómo está siendo hecho el mundo, una imagen que de hecho legitima su propia producción. Todas las representaciones se ajustaban al proyecto civilizatorio moderno en el que indígenas y negros aparecían como comunidades "atrasadas" que debían iniciar el camino hacia el progreso y la civilización bajo la tutela europea.

En este tipo de sistemas de producción del conocimiento se iguala la representación del mundo con su lugar de espacialización. Para entender mejor esto me detendré en los aportes de Castro-Gómez.

\section{(T2) De la localización de los saberes}

Santiago Castro-Gómez, en su libro La hybris del punto cero, se propone pensar la matriz poder/conocimiento en las prácticas coloniales de los siglos XVIII y XIX, en la Nueva Granda. Para ellos, empieza con una reflexión sobre los lugares de la llustración en la que, en sintonía con Massey, sostiene que la Ilustración no es un fenómeno europeo que después se difunde por todo el mundo, sino que es "un conjunto de discursos con diferentes lugares de producción y enunciación que gozaban ya en el siglo XVIII de una circulación mundial" (Castro-Gómez, 2005a, p. 22). A partir de esta tesis, el autor considera el proyecto ilustrado moderno desde la categoría del punto cero. Para Castro-Gómez (2005a), la ciencia moderna tenía la pretensión de 
poder observar sus objetos de estudios desde un punto inobservable de observación que garantizaba la absoluta objetividad del conocimiento (para llegar a este punto cero de observación era necesario prescindir de todas las determinaciones particulares del observador, esto es, su raza, su género, su lugar de enunciación, etc.); esta pretensión de deslocalizarlas enunciaciones científicas en aras de la objetividad es lo que nuestro autor llama la hybris del punto cero:

Los habitantes del punto cero (científicos y filósofos ilustrados) están convencidos de que pueden adquirir un punto de vista sobre el cual no es posible adoptar ningún punto de vista... Los griegos decían que la hybris es el peor de los pecados, pues supone la ilusión de poder rebasar los límites propios de la condición mortal y llegar a ser como los dioses. La hybris supone entonces el desconocimiento de la espacialidad y es por ello un sinónimo de arrogancia y desmesura (Castro-Gómez, 2005a, pp. 18-19).

Así, los pensadores ilustrados, al pretender carecer de un lugar de enunciación y traducción, serían culpables del pecado de la hybris.

Pero este punto cero no funciona sólo en un plano epistemológico. Al ser parte constitutiva de la producción de discursos, el punto cero articula prácticas reales de dominación y control.

El punto cero es el del comienzo epistemológico absoluto, pero también el del control económico y social sobre el mundo. Ubicarse en el punto cero equivale a tener el poder de instituir, de representar, de construir una visión sobre el mundo social y natural reconocida como legítima y avalada por el Estado (Castro-Gómez, 2005a, p. 25).

Esto es evidente en la colonización europea que tematiza desde esta perspectiva el grupo de estudios poscoloniales.

En Orientalismo, Edward Said, uno de los mayores representantes del poscolonialismo asiático, "plantea de forma clara que el proyecto ilustrado de la ciencia del hombre se sustenta en un imaginario geopolítico (el occidentalismo) que postula la superioridad de la raza blanca europea sobre todas las demás formas culturales del planeta" (Castro-Gómez, 2005a, p.43). Desde 
la argumentación de Castro-Gómez, esto tiene un trasfondo discursivo en los relatos bíblicos que empezaron a funcionar en la llustración como legitimadores del proyecto colonizador. Según explica Castro-Gómez (2005a):

El cristianismo reinterpretó la antigua división jerárquica del mundo... Las tres regiones geográficas [del Viejo Mundo: África, Asia y Europa] eran vistas como el lugar donde se asentaron los tres hijos de Noé después del diluvio y, por tanto, como habitadas por tres tipos completamente distintos de gente. Los hijos de Sem poblaron Asia, los de Cam se establecieron en África y los de Jafet se asentaron en Europa. Esto quiere decir que las tres partes del mundo conocido fueron ordenadas jerárquicamente según un criterio de diferenciación étnica: los asiáticos y los africanos, descendientes de aquellos hijos que según el relato bíblico cayeron en desgracia frente a su padre, eran tenidos como racial y culturalmente inferiores a los europeos, descendientes directos de Jafet, el hijo amado de Noé (p. 55).

La división en zonas geográficas de las culturas ancestrales dejaba por fuera a la recién descubierta América; las representaciones europeas de esta división del mundo hicieron corresponder al continente americano con la descendencia de Jafet, esto es de Europa, lo que daba a los europeos el legítimo derecho y hasta la obligación divina de colonizar a los pueblos americanos. Luego, la colonización no es un fenómeno accesorio de la modernidad, sino su otro constitutivo. La teoría poscolonial sostiene como tesis fundamental que la modernidad y la colonialidad no son fenómenos sucesivos en el tiempo, sino que son simultáneos en el espacio (Castro-Gómez, 2005b). Modernidad y colonialidad son caras de la misma moneda, según aducen los teóricos latinoamericanos del poscolonialismo.

Según Enrique Dussel, poscolonialista, el mito eurocéntrico de la modernidad surge con el descubrimiento de América. Es un discurso sobre los orígenes del pensamiento ilustrado que piensa la modernidad como un fenómeno exclusivamente europeo, que luego se difundió por todo el mundo; es un discurso que sólo puede emerger ante la producción de otro colonial. CastroGómez (2005a) refiere: 
De acuerdo con esta visión, Europa posee cualidades internas únicas que le habrían permitido desarrollar la racionalidad científico-técnica, lo cual explica la superioridad de su cultura sobre todas las demás. De este modo, el mito eurocéntrico de la modernidad sería la pretensión que identifica la particularidad europea con la universalidad sin más (p. 49).

Según el filósofo colombiano, la importancia de pensar la modernidad como un fenómeno mundial (lo que Dussel Ilama el "paradigma planetario") radica en que nos permite evidenciar la coexistencia de lugares desde los que la Ilustración es enunciada. Desde esta perspectiva, los discursos no viajan desde el centro hasta la periferia (en la que son recibidos y asimilados), sino que circulan por todo el sistema-mundo, anclándose en diferentes nodos de poder (Castro-Gómez, 2005a. p. 50).

Es desde entonces que se constituye una geografía del conocimiento, que define jerarquías a partir de los lugares de producción de conocimiento y que genera un sistema particular de enunciación, el cual mantiene y controla las funciones de centro y periferia en las representaciones. Categorías como primitivo, bárbaro, tradicional, étnico, entre otras, definen un lugar periférico de enunciación que queda invalidado para la producción de conocimiento científico.

De este modo, las muchas formas de conocer están ubicadas en una concepción de la historia que deslegitima su coexistencia espacial y las ordena de acuerdo a un esquema teleológico de progresión temporal. Las diversas formas de conocimiento desplegadas por la humanidad conducirán paulatinamente hacia una única forma legítima de conocer el mundo; la desplegada por la racionalidad científico-técnica de la modernidad (Castro-Gómez, 2005a, p. 45).

A esta clasificación geográfica para la jerarquización de los saberes asistimos aún hoy. En seguida reflexionaremos sobre este punto.

\section{Geopolíticas del conocimiento}

Uno de los grandes aportes de Foucault que retoman los estudios poscoloniales fue hacernos ver cómo los sistemas expertos de representación están 
vinculados con geopolíticas de poder y la manera como el conocimiento ayudó a la consolidación hegemónica de los sistemas representacionales (Castro-Gómez, 1998, p. 169). Las instituciones académicas hegemónicas producen discursos y representaciones sobre el otro que justifican políticas de subordinación de las "periferias", construyendo discursivamente una imagen de las culturas que legitima las divisiones binarias ya nombradas y la tutela de los países "superiores" sobre aquellos que son estudiados por ellos.

La academia internacional y sus teorizaciones sobre el otro funcionan como afirmación de una serie de diferenciaciones que siguen administrando las funciones de centro y periferia dentro del sistema de enunciación. La concentración de medios y recursos otorgan a esta academia una "investidura" de Centro que, apelando a alteridades, marginalidades, subalternidades, legitima su facultad simbólica y representativa. Dice Richard (1998): "la autoridad teórica de la función-centro reside en ese monopolio del poder-derepresentación según el cual,'representar'es controlar los medios discursivos que subordinan el objeto de saber a una economía conceptual declarada superior" (p. 250).

Estas jerarquías que se instauran actualmente en el marco de la globalidad habilitan a la academia-centro para patentar los códigos de figuración teórica que otorgarán legitimidad a sus objetos de estudio y a las representaciones sobre estos (Richard, 1998, p. 255). Las representaciones periféricas (aquellas que se producen por fuera del centro) deben justificar sus "efectos de verdad" ante la particular "política de interpretación" impuesta por la academia hegemónica (el centro). Estas políticas de interpretación y de representación refuerzan la generación discursiva (que se extiende a planos reales) de ámbitos de exterioridad que insisten en la idea de categorizaciones binarias, vigente aún en los aparatos metropolitanos de producción del saber (CastroGómez, 1998, p. 173).

En todo este análisis es de vital importancia recordar que estas jerarquías están basadas en delimitaciones geográficas en las que las determinaciones espaciales están subordinadas a un ordenamiento temporal de las poblaciones y las culturas (representadas como desarrolladas o subdesarrolladas, civilizadas o primitivas, etc.). 
En este marco es pertinente ampliar la categoría de Massey de geografía de la producción del conocimiento a geopolíticas del conocimiento, categoría ampliamente utilizada por Walter Mignolo, teórico poscolonial argentino. Decía más arriba con Castro-Gómez (2005a) que una de las consecuencias de la hybris del punto cero es "la invisibilización del lugar particular de enunciación para convertirlo en un lugar sin lugar, en un universal" (p. 61). Esta pretensión de universalidad de los discursos modernos está íntimamente relacionada con sistemas de dominación como hemos señalado varias veces; en palabras de Castro-Gómez (2005a):

Esta tendencia a convertir la historia local en un diseño global, corre paralela al establecimiento de ese lugar particular como centro de poder geopolítico. A la centralidad de España, luego de Francia, Holanda, Inglaterra y los Estados Unidos en el sistema-mundo, corresponde la pretensión de convertir su propia historia local en lugar único y universal de enunciación y de producción de conocimientos. Los conocimientos que no se produzcan en esos centros de poder o en los circuitos controlados por ellos, son declarados irrelevantes y 'precientíficos' (p. 61).

En este sentido, la historización de los discursos de la modernidad, así como su espacialización, contribuyen al intento de afirmar la multiplicidad epistémica que supone la legitimación intrínseca de toda producción discursiva y que abre la posibilidad de pensar otros paradigmas epistemológicos y, desde allí, mundos otros y realidades otras.

\section{Referencias}

Castro-Gómez, S. (1998). Latinoamericanismo, Modernidad, Globalización. Prolegómenos a una Crítica Poscolonial de la Razón. En S. Castro-Gómez y E. Mendieta (Eds.), Teorías sin disciplina. Latinoamericanismo, poscolonialidad y globalización en debate (pp. 169-205). México: University of San Francisco.

Castro-Gómez, S. (2005a) La hybris del punto cero. Ciencia, raza ellustración en la Nueva Granada (1750-1816). Bogotá, Colombia: Pontificia Universidad Javeriana. 
Castro-Gómez, S. (2005b). La poscolonialidad explicada a los niños. Popayán, Colombia: Universidad del Cauca.

Foucault, M. (1968) Las palabras y las cosas. Una arqueología de las ciencias humanas (E. C. Frost, Trad.). México: Siglo XXI.

Foucault, M. (1970) La arqueología del saber(A. Garzón del Camino, Trad.). México: Siglo XXI.

Foucault, M. (1984, octubre).De los Espacios Otros (P. Blitstein y T. Lima, Trads.). [Versión electrónica]. Architecture, Mouvement, Continuité, (5). Recuperado en febrero de 2009 de http://www.scribd.com/doc/4650039/ Foucault-M-De-los-espacios-otros?autodown=doc

Foucault, M. (1992). El orden del discurso (A. González Troyano, Trad.). Buenos Aires, Argentina:Tusquets.

Foucault, M. (2003). ¿Qué es la Crítica? (J.de la Higuera, Trad.). En Sobre la Ilustración (pp. 3-52). Madrid, España:Tecnos.

Massey, D. (2005). For space.London, UK: Sage publications.

Richard, N. (1998) Intersectando Latinoamérica con el Latinoamericanismo: Discurso Académico y Crítica Cultural. En S. Castro-Gómez y E. Mendieta (Eds.), Teorías sin disciplina. Latinoamericanismo, poscolonialidad y globalización en debate (pp. 245 - 270). México: University of San Francisco. 